\title{
Weak-field approximation of effective gravitational theory with local Galilean invariance 1
}

\author{
R. R. Cuzinatto ${ }^{a}$, P. J. Pompeia ${ }^{a, b, c}$, M. de Montigny ${ }^{a, d}$, F. C. \\ Khanna $^{a, e}$ \\ ${ }^{a}$ Theoretical Physics Institute, University of Alberta, \\ Edmonton, Alberta, Canada T6G 2J1 \\ ${ }^{b}$ Instituto de Física Teórica, Universidade Estadual Paulista, \\ Rua Pamplona 145, 01405-000, São Paulo, SP, Brazil \\ ${ }^{c}$ Comando-Geral de Tecnologia Aeroespacial, Instituto de Fomento e \\ Coordenação Industrial, \\ Praça Mal. Eduardo Gomes 50, 12228-901, São José dos Campos, SP, \\ Brazil \\ ${ }^{d}$ Campus Saint-Jean, University of Alberta, \\ Edmonton, Alberta, Canada T6C 4G9 \\ ${ }^{e}$ TRIUMF, 4004, Westbrook Mall, \\ Vancouver, British Columbia, Canada V6T $2 A 3$
}

\begin{abstract}
We examine the weak-field approximation of locally Galilean invariant gravitational theories with general covariance in a $(4+1)$ dimensional Galilean framework. The additional degrees of freedom allow us to obtain Poisson, diffusion, and Schrödinger equations for the fluctuation field. An advantage of this approach over the usual $(3+1)$ dimensional General Relativity is that it allows us to choose an ansatz for the fluctuation field that can accommodate the field equations of the Lagrangian approach to MOdified Newtonian Dynamics (MOND) known as AQUAdratic Lagrangian (AQUAL). We investigate a wave solution for the Schrödinger equations.
\end{abstract}

Keywords: Galilean invariance; general relativity; weak-field approximation PACS: 11.30.-j; 11.30.Cp; 04.25.-g; 04.25.Nx

\footnotetext{
${ }^{1}$ rcuzin@phys .ualberta.ca,pedro.pompeia@gmail.com,montigny@phys . ualberta.ca, khanna@phys .ualberta.ca
} 


\section{Introduction to the Galilean framework}

It is well known that the Galilei group describes non-relativistic, or lowenergy, phenomena [1]. The study of non-relativistic limits of various relativistic phenomena is not always a straightforward procedure; for instance, the straightforward low-velocity approximation of electromagnetism may lead to one limit, whereas there exist in fact two limits of electromagnetism [2, 3. Another example of such a subtlety is the concept of spin; although historically explained within the context of the relativistic Dirac equation, spin can also be understood in terms of the Galilean theory, so that it is not a purely relativistic notion [4].

The Galilean symmetry is, in some respects, more intricate than the Poincaré symmetry, which underlies relativistic physics [1. For instance, Galilean systems are not usually described in terms of tensors, because of the absence of a metric. This problem was circumvented, for flat manifolds, with the formalism of Galilean covariance, originally examined by various authors [5]-7]. This formalism consists in describing Galilean theories in a tensorial form by means of a light-cone metric defined on a $(4+1)$-dimensional Minkowski manifold. As mentioned in these early papers, the reduction from this extended Minkowski space to a $(3+1)$-dimensional spacetime can lead to the Galilean invariant theory as well as the Minkowski theory, thereby providing a unified framework to treat both the Galilei and the Lorentz kinematics in a simple and elegant manner [6]-7].

In this paper, we extend the formalism to the Riemannian geometry underlying gravitational models, following the lines of Ref. [8]. One motivation for this work is the fact that General Relativity (GR) does not provide satisfactory explanations to some gravitational phenomena involving nonrelativistic objects, e.g. the dark matter and dark energy problems [9]. The presence of dark matter is required in order to explain, for instance, the rotation curves of spiral galaxies, and dark energy appears in the context of cosmology in order to describe the accelerated expansion of the universe. Although quite diverse, these issues present a common feature: they occur in the low-energy regime. This indicates that local Galilean invariance as the underlying symmetry in the theory of gravitation could shed new light on these questions. The MOdified Newtonian Dynamics (MOND) approach [10]-13] addresses these issues either by modifying the law of inertia or by altering the Newtonian theory of gravity phenomenologically. Our perspective is aligned with the latter approach; we will show that it accommodates the AQUAL fundamental equation in a rather natural way, with the extra dimension being related to the mass density. 
The Galilean formalism, described in Refs. [5]-[7], consists in building Lorentz-covariant action functionals defined on a $(4+1)$-dimensional Minkowski manifold. This manifold is described in terms of the Galilean five-vector, $\left(\mathrm{x}, x^{4}, x^{5}\right)$, which transforms under Galilei boosts, with relative velocity $\mathbf{v}$, as

$$
\begin{aligned}
\mathbf{x}^{\prime} & =\mathbf{x}-\mathbf{v} x^{4} \\
x^{\prime 4} & =x^{4} \\
x^{\prime 5} & =x^{5}-\mathbf{v} \cdot \mathbf{x}+\frac{1}{2} \mathbf{v}^{2} x^{4} .
\end{aligned}
$$

Thus, general Galilean transformations (including rotations, Galilei boosts and translations) have a form similar to the usual Poincaré transformations,

$$
x^{\prime \mu}=\Lambda_{\nu}^{\mu} x^{\nu}+a^{\nu}, \quad \mu, \nu=1, \ldots, 5 .
$$

The Galilei algebra of a $(3+1)$-dimensional space-time is an 11-dimensional subalgebra of the 15-dimensional Lie algebra isomorphic to the Poincaré algebra in $(4+1)$-dimensional spacetime.

The tensor methods utilized in the Galilean covariance formalism are based on the scalar product, $A \cdot B=\mathbf{A} \cdot \mathbf{B}-A_{4} B_{5}-A_{5} B_{4}$, which is invariant under the transformations in Eq. (11). This suggests to employ, on a locally flat manifold, the following Galilean metric:

$$
\eta_{\mu \nu}=\left(\begin{array}{ccc}
\mathbf{1}_{3 \times 3} & 0 & 0 \\
0 & 0 & -1 \\
0 & -1 & 0
\end{array}\right) .
$$

We call Galilean manifold this 5-dimensional flat manifold which, although equivalent to a Minkowski manifold in $(4+1)$ dimensions, reduces to the Galilean space-time via appropriate ansätze. The five-momentum, $p_{\mu}=$ $\mathrm{i} \partial_{\mu}=\left(\mathrm{i} \nabla, \mathrm{i} \partial_{t}, \mathrm{i} \partial_{S}\right)=\left(\mathbf{p}, \frac{E}{c}, m c\right)$, where $c$ is a parameter with the dimensions of velocity, and $S$ is the extra coordinate, suggests that the additional coordinate, $x^{5}=\frac{S}{c}$, may be seen as the conjugate of $m c$, where $m$ is the mass.

In this paper, we utilize the Galilean manifold to examine the weak-field approximation and we obtain Galilean differential equations through the procedures that usually describe gravitational waves in GR. This will result in Poisson, diffusion, and Schrödinger equations for the fluctuation field. We shall see in Section 3 that it is possible to accommodate MOND [10]-[13] within the Galilean gravity. Henceforth, we consider a 5-dimensional Riemannian spacetime; that is, a 5-dimensional differential manifold where an invertible symmetric metric tensor is defined. Thus we can define quantities usually encountered in general relativity: a symmetric connection, given by 
the Christoffel symbols; covariant derivatives; curvature, or Riemann tensor; Ricci tensor, and the like. The main advantage of the Galilean covariance method is that these quantities have a form similar to GR, but lead to Galilei-invariant expressions through a natural definition of the ansätze.

In Section 2, we consider a Lagrangian which produces equations that are linear in the second-order derivative of the metric. We also discuss the weak-field approximation with local Galilean invariance, and obtain field equations. We reduce the number of degrees of freedom in the perturbation field by using the trace-reverse perturbation tensor and gauge-fixing conditions. These results are employed in Section 3, where we examine three ansätze: the perturbation field does not depend on $x^{5}$, which leads to a Poisson equation (Section 3.1); the complex ansatz, which provides a Schrödinger equation (Section 3.2); and the real ansatz, which gives a diffusion equation (Section 3.3). The ansatz of Section 3.1 may lead to the AQUAdratic Lagrangian (AQUAL) field equation for MOND theory, and the anstze in Section 3.3 suggest a different version for the AQUAL field equation. We also discuss solutions of the Schrödinger equation in Section 3.2. We make concluding remarks in Section 4 .

\section{Weak-field approximation in Galilean gravity}

Typically, the Lagrangian that leads to the field equations for the pointdependent metric $g_{\mu \nu}$ is separated into two parts: $\mathcal{L}_{0}$, which depends on the metric, and $\mathcal{L}_{\text {matter }}$, which describes a matter field. The variational principle leads to [14, 15]

$$
\frac{\delta \mathcal{L}_{0}}{\delta g_{\mu \nu}}=-\frac{\sqrt{-g}}{2} T^{\mu \nu}, \quad T^{\mu \nu} \equiv \frac{2}{\sqrt{-g}} \frac{\delta \mathcal{L}_{\text {matter }}}{\delta g_{\mu \nu}}
$$

where $g \equiv \operatorname{det}\left(-g_{\mu \nu}\right)$, and $T^{\mu \nu}$ is the mass-energy-momentum tensor. Henceforth, greek indices, $\mu, \nu$, etc. run from 1 to 5 , whereas latin indices, $j, k$, etc. denote the usual three spatial components.

Firstly, we consider the gravitational Lagrangian to be the EinsteinHilbert Lagrangian,

$$
\mathcal{L}_{0}=\sqrt{-g} R
$$

where $R$ is the scalar curvature. This Lagrangian leads to Einstein's equations [14],

$$
G_{\mu \nu} \equiv R_{\mu \nu}-\frac{1}{2} g_{\mu \nu} R=\kappa T_{\mu \nu}
$$


where

$$
\kappa=8 \pi G,
$$

and $G$ is the gravitational constant. Note that we work with $c=1$.

The following relations are equivalent to Eq. (4):

$$
\begin{aligned}
& R_{\mu \nu}=\kappa S_{\mu \nu}, \\
& S_{\mu \nu}=T_{\mu \nu}-\frac{1}{3} g_{\mu \nu} T,
\end{aligned}
$$

where $T=T_{\mu}^{\mu}$. Note that the factor $1 / 3$, instead of the usual $1 / 2$, follows from the trace of Eq. (4) which leads to $R_{\mu \nu}=\kappa\left(T_{\mu \nu}-g_{\mu \nu} \frac{1}{D-2} T\right)$, with $D=5$ instead of $D=4$.

The weak-field, or linearized-gravity, approximation is described by the perturbation, or fluctuation, tensor field $h_{\mu \nu}$, defined by

$$
g_{\mu \nu}=\eta_{\mu \nu}+h_{\mu \nu},
$$

where $\left|h_{\mu \nu}\right|<<1$. Thus $h_{\mu \nu}$ is the first-order correction to the Galilean metric, $\eta_{\mu \nu}$ in Eq. (3). The curved spacetime described by $g_{\mu \nu}$ is a perturbation of the flat $(4+1)$-dimensional Minkowski manifold.

Consider a finite, global (i.e. $x$-independent) Galilean transformation, in the form of Eq. (2),

$$
x^{\mu} \rightarrow x^{\prime \mu}=\Lambda_{\nu}^{\mu} x^{\nu}+a^{\mu},
$$

where $\Lambda_{\rho}^{\mu} \Lambda_{\sigma}^{\nu} \eta_{\mu \nu}=\eta_{\rho \sigma}$, and $\eta_{\mu \nu}$ is the flat-space metric given in Eq. (3). The metric transforms as

$$
\begin{aligned}
g^{\prime}{ }_{\mu \nu} & =\frac{\partial x^{\rho}}{\partial x^{\prime \mu}} \frac{\partial x^{\sigma}}{\partial x^{\prime \nu}} g_{\rho \sigma}=\left(\Lambda^{-1}\right)_{\mu}^{\rho}\left(\Lambda^{-1}\right)^{\sigma}{ }_{\nu} g_{\rho \sigma} \\
& =\Lambda_{\mu}^{\rho} \Lambda_{\nu}^{\sigma}\left(\eta_{\rho \sigma}+h_{\rho \sigma}\right)=\eta_{\mu \nu}+\Lambda_{\mu}^{\rho} \Lambda_{\nu}^{\sigma} h_{\rho \sigma} .
\end{aligned}
$$

We have used the property $\left(\Lambda^{-1}\right)^{\rho}{ }_{\nu}=\Lambda_{\nu}{ }^{\rho}$.

Since we have, in the new frame, $g^{\prime}{ }_{\mu \nu}\left(x^{\prime}\right)=\eta_{\mu \nu}+h^{\prime}{ }_{\mu \nu}\left(x^{\prime}\right)$, we observe that $h_{\mu \nu}$ transforms like a tensor under Galilean transformations,

$$
h^{\prime}{ }_{\mu \nu}=\Lambda_{\mu}^{\rho} \Lambda_{\nu}^{\sigma} h_{\rho \sigma} .
$$

From Eq. (44), we find

$$
\begin{aligned}
G_{\mu \nu} & =\frac{1}{2}\left[2 \partial_{\rho} \partial_{(\mu} h_{\nu)}^{\rho}-\partial_{\mu} \partial_{\nu} h-\square h_{\mu \nu}-\eta_{\mu \nu}\left(\partial_{\rho} \partial_{\sigma} h^{\rho \sigma}-\square h\right)\right] \\
& =\kappa T_{\mu \nu},
\end{aligned}
$$


where the indices between parentheses are symmetrized; that is, $A_{(\mu \nu)}=$ $\frac{1}{2}\left(A_{\mu \nu}+A_{\nu \mu}\right)$.

We find, from Eq. (6) , the equivalent result:

$$
\kappa S_{\mu \nu}=\frac{1}{2}\left[2 \partial_{\rho} \partial_{(\mu} h_{\nu)}^{\rho}-\partial_{\mu} \partial_{\nu} h-\square h_{\mu \nu}\right] .
$$

Since $R$ is of first order in $h_{\mu \nu}, T_{\mu \nu}$ (or $S_{\mu \nu}$ ) must also be of first order. This explains why we use $\eta_{\mu \nu}$, instead of $g_{\mu \nu}$, in Eq. (8) (or Eq. (9)). In this approximation, the interpretation of the components of the mass-energymomentum tensor is manifest. As explained in Ref. [3], the components $T_{i j}$ are the density of momentum flux of the matter field; $T_{5 j}$ is the field momentum density; $T_{4 j}$ is the density of energy flux; $-T_{45}$ the energy density, and $-T_{55}$ is the density of mass of the field. The component $T_{44}$ does not have a clear physical interpretation and it seems to be simply a remnant of the extended manifold.

In order to obtain a formal solution of the field equations, let us work with the harmonic gauge condition (see Section 7.4 of Ref. [14]):

$$
g^{\mu \nu} \Gamma_{\mu \nu}^{\rho}=\frac{1}{2} \eta^{\rho \sigma}\left(2 \partial_{\nu} h_{\sigma}^{\nu}-\partial_{\sigma} h\right)=0 .
$$

If we substitute this into the linearized field equation, Eq. (9), and then apply a Fourier transform, we obtain

$$
\kappa S_{\mu \nu}(p)=\frac{1}{2} p_{\rho} p^{\rho} h_{\mu \nu}(p) .
$$

By solving Eq. (10) for $h_{\mu \nu}(p)$, and performing an inverse Fourier transform, we obtain

$$
h_{\sigma \tau}(x)=2 \kappa \int d^{5} x^{\prime} G\left(x-x^{\prime}\right) S_{\sigma \tau}\left(x^{\prime}\right),
$$

where

$$
G\left(x-x^{\prime}\right)=\int d^{5} p e^{\mathrm{i} p_{\rho}\left(x-x^{\prime}\right)^{\rho}} \frac{1}{p^{2}}=\int d^{5} p e^{\mathrm{i} p_{\rho}\left(x-x^{\prime}\right)^{\rho}} \frac{1}{\left(\mathbf{p}^{2}-2 p_{4} p_{5}\right)} .
$$

Note that this propagator was used in a different context in Ref. [16]. By integrating this expression for the Green's function, we obtain the field components as integrals of the energy-momentum tensor, Eq. (11).

For later convenience, let us define the trace-reverse perturbation tensor:

$$
\hat{h}_{\mu \nu} \equiv h_{\mu \nu}-\frac{1}{2} \eta_{\mu \nu} h
$$


so that the terms involving the trace of $\hat{h}_{\mu \nu}$ do not appear in the field equation. Then we can write Eq. (8), i.e. the field equations without gauge fixing, as follows:

$$
\kappa T_{\mu \nu}=\partial_{\rho} \partial_{(\mu} \hat{h}_{\nu)}^{\rho}-\frac{1}{2} \eta_{\mu \nu} \partial_{\rho} \partial_{\sigma} \hat{h}^{\rho}-\frac{1}{2} \square \hat{h}_{\mu \nu} .
$$

Furthermore, with the Lorenz gauge condition, $\partial_{\rho} \hat{h}_{\nu}{ }^{\rho}=0$, we obtain the general field equations

$$
\kappa T_{\mu \nu}=-\frac{1}{2}\left[\nabla^{2}-2 \partial_{4} \partial_{5}\right] \hat{h}_{\mu \nu} .
$$

This equation takes various forms, depending on different hypotheses concerning the functional form of the field $\hat{h}_{\mu \nu}$. Specific ansätze are discussed in Section 3 ,

(About the gauge fixing, let us comment that, on the $(4+1)$-dimensional manifold, the rank-2 symmetric tensor $\hat{h}_{\mu \nu}$ comprises 15 independent components. The Lorenz condition, $\partial_{\rho} \hat{h}_{\nu}{ }^{\rho}=0$, introduces five more constraints, and the choice of coordinate system implies five additional restrictions. Thus the linearized tensor has only five independent components: $\hat{h}_{1 i}, \hat{h}_{23}, \hat{h}_{33}$. This will be transparent in Eq. (27) of Section 3.2, when we discuss the solutions of the Schrödinger equation. In discussions of gravitational waves in five-dimensional GR, one obtains the same number of independent components for $h_{\mu \nu}$ (for instance, see Eq. (6) in Ref. [18]). Let us observe that the harmonic gauge condition and the Lorentz gauge condition are equivalent; subsequently, we choose to use one or the other at convenience.)

\section{Three ansätze for the perturbation field}

Hereafter we examine three specifc ansätze of $\hat{h}_{\mu \nu}$ as a function of $x^{5}$, and we examine Eq. (14), in the presence of matter-energy fields.

\subsection{Poisson equation}

If $\hat{h}_{\mu \nu}$ does not depend on $x^{5}$, so that

$$
\hat{h}_{\mu \nu}=\hat{\mathfrak{h}}_{\mu \nu}\left(\mathbf{x}, x^{4}\right),
$$

we see that Eq. (14) reduces to the Poisson equation:

$$
\nabla^{2} \hat{h}_{\mu \nu}\left(\mathbf{x}, x^{4}\right)=-2 \kappa T_{\mu \nu} .
$$


In vacuum, $T_{\mu \nu}=0$, it simply becomes the Laplace equation. Notice that, from the point of view of the Galilean approach, this ansatz means that we are considering $h_{\mu \nu}$ as being a massless field.

We already mentioned in Section 2 that $-T_{55}$ is the mass density,

$$
-T_{55}=\rho .
$$

Then, with Eq. (17) and

$$
\hat{h}_{55}=4 \phi_{N},
$$

we find that Eq. (16) becomes the Newton's gravitational law:

$$
\nabla^{2} \phi_{N}=4 \pi G \rho
$$

where $\phi_{N}$ is the Newtonian potential, and $\kappa$ is defined in Eq. (5).

Therefore, if we keep Eq. (17), but, instead of Eq. (18), we consider $\hat{h}_{55}$ given by

$$
\nabla \hat{h}_{55}=4 \mu(x) \nabla \phi, \quad x \equiv|\nabla \phi| / a_{0}
$$

then Eq. (16) gives the Poisson equation utilized in the AQUAL formalism [12, 13]:

$$
\nabla \cdot[\mu(x) \nabla \phi]=4 \pi G \rho .
$$

The Milgrom's transition function $\mu$ depends only on the first derivative of the field $\phi$, which is the gravitational potential that drives the motion; that is, $\mathbf{a}=-\nabla \phi$. According to MOND, bodies subjected to gravitational force that move with an acceleration smaller than $a_{0}$ should have a dynamic behaviour different from Newtonian mechanics. AQUAL reproduces exactly the MOND formula, $\mu(x) \mathbf{a}=-\nabla \phi_{N}$, where $\phi_{N}$ is the Newtonian gravitational potential. In MOND models, $\mu$ can, for instance, consist of the "standard function", $\mu(x)=x / \sqrt{1+x^{2}}$, with $x=|\mathbf{a}| / a_{0}$, which exhibits the additional fundamental constant of the theory: the modulus of the acceleration, $a_{0} \sim 10^{-10} \mathrm{~m} / \mathrm{s}^{2}$. For the standard $\mu$-function given above in the large- $\frac{|\nabla \phi|}{a_{0}}$ limit, we expand $\nabla h_{55}$ as follows:

$$
\begin{aligned}
\nabla h_{55} & \simeq \frac{4 \nabla \phi}{\sqrt{1+\left(a_{0} /|\nabla \phi|\right)^{2}}} \\
& =4 \nabla \phi\left[1-\frac{1}{2}\left(\frac{a_{0}}{|\nabla \phi|}\right)^{2}+\frac{3}{8}\left(\frac{a_{0}}{|\nabla \phi|}\right)^{4}-\frac{5}{16}\left(\frac{a_{0}}{|\nabla \phi|}\right)^{6}+\cdots\right],
\end{aligned}
$$

which approaches the Newtonian limit when $a_{0}<<|\nabla \phi|$. In this limit we can approximate $h_{55}$ by

$$
h_{55}=4 \mu(x) \phi
$$


since $\nabla \mu(x)=\frac{d \mu}{d x} \frac{1}{a_{0}|\nabla \phi|}(\nabla \phi \cdot \nabla) \nabla \phi$, and $\lim _{x \rightarrow \infty} \frac{d \mu}{d x}=\lim _{x \rightarrow \infty} \frac{1}{\sqrt{\left(1+x^{2}\right)^{3}}}=$ 0 , so that $\nabla h_{55}=4 \mu(x) \nabla \phi+4 \phi \nabla \mu(x) \simeq 4 \mu(x) \nabla \phi$. This identification cannot be done in the small- $\frac{|\nabla \phi|}{a_{0}}$ limit, where we would obtain

$$
\begin{aligned}
\nabla h_{55} & =\frac{4 \nabla \phi|\nabla \phi| / a_{0}}{\sqrt{1+\left(|\nabla \phi| / a_{0}\right)^{2}}} \\
& =4 \nabla \phi\left[\left(\frac{|\nabla \phi|}{a_{0}}\right)-\frac{1}{2}\left(\frac{|\nabla \phi|}{a_{0}}\right)^{3}+\frac{3}{8}\left(\frac{|\nabla \phi|}{a_{0}}\right)^{5}-\frac{5}{16}\left(\frac{|\nabla \phi|}{a_{0}}\right)^{7}+\cdots\right],
\end{aligned}
$$

which approaches the Newtonian limit when $|\nabla \phi|<<a_{0}$. In this case $h_{55}$ cannot be integrated and in order to obtain consistency with the AQUAL theory we should choose

$$
\nabla \hat{h}_{55} \simeq 4 \mu(x) \nabla \phi+\nabla \times \mathbf{f}
$$

where $\mathbf{f}$ is a function to be determined. This is in accordance to what is expected from the AQUAL version of MOND where the theory is determined up to a curl of an arbitrary vector field [13].

The same can be done for the "simple function", $\mu(x)=x /(1+x)$. In the small- $\frac{|\nabla \phi|}{a_{0}}$ limit, we write $\nabla h_{55}$ as

$$
\begin{aligned}
\nabla h_{55} & =\frac{4 \nabla \phi|\nabla \phi| / a_{0}}{1+|\nabla \phi| / a_{0}} \\
& =4 \nabla \phi\left[\left(\frac{|\nabla \phi|}{a_{0}}\right)-\left(\frac{|\nabla \phi|}{a_{0}}\right)^{2}+\left(\frac{|\nabla \phi|}{a_{0}}\right)^{3}-\left(\frac{|\nabla \phi|}{a_{0}}\right)^{4}+\cdots\right]
\end{aligned}
$$

which approaches zero in the limit $|\nabla \phi|<<a_{0}$. In this case, the $h_{55}=\mu(x) \phi$ is also valid in first order of approximation. The simple $\mu$-function, in the large $-\frac{|\nabla \phi|}{a_{0}}$ limit, is obtained by expanding $\nabla h_{55}$ as

$$
\begin{aligned}
\nabla h_{55} & =\frac{4 \nabla \phi}{1+a_{0} /|\nabla \phi|} \\
& =4 \nabla \phi\left[1-\frac{a_{0}}{|\nabla \phi|}+\left(\frac{a_{0}}{|\nabla \phi|}\right)^{2}-\left(\frac{a_{0}}{|\nabla \phi|}\right)^{3}+\cdots\right]
\end{aligned}
$$

which approaches the Newtonian limit when $a_{0}<<|\nabla \phi|$. Here a curl of a vector field also should be added in order to integrate $h_{55}$.

\subsection{Schrödinger equation and its wave solutions}

We now turn to the case where the gravitational perturbation is a massive field. Since $p_{5}$ is an invariant of the Galilean algebra [3, 16], the natural ansatz is given by

$$
\hat{h}_{\mu \nu}=e^{-\mathrm{i} m x^{5}} \hat{\mathfrak{h}}_{\mu \nu}\left(\mathbf{x}, x^{4}\right) .
$$


A similar situation occurs in the Galilean analyzes of the Proca field [3]. In fact, if we follow our recent paper [17], and consider both the positive and negative mass contributions to Eq. (21), it amounts to adding the complex conjugate to Eq. (21), so that $\hat{h}_{\mu \nu}$ is real. This is analogous to the treatment of plane waves discussed in Section 10.2 of [14]. The physical field $\hat{\mathfrak{h}}_{\mu \nu}\left(\mathbf{x}, x^{4}\right)$ should be real as well. With $\hat{h}_{\mu \nu}$ defined as above, we see that Eq. (14) becomes

$$
-2 \kappa T_{\mu \nu}=\nabla^{2} \hat{h}_{\mu \nu}+2 m \mathrm{i} \partial_{4} \hat{h}_{\mu \nu}
$$

This is an inhomogeneous Schrödinger equation for each tensor component.

Next, we find a solution for the 'free-particle' situation, or, equivalently, the vacuum case, $T_{\mu \nu}=0$. This leads to the Schrödinger equation,

$$
\mathrm{i} \partial_{4} \hat{h}_{\mu \nu}=-\frac{1}{2 m} \nabla^{2} \hat{h}_{\mu \nu} .
$$

If we write the complex ansatz of Eq. (21) for $\hat{h}_{\mu \nu}$ in the form,

$$
\hat{h}_{\mu \nu}=e^{-\mathrm{i} m x^{5}} T\left(x^{4}\right) X_{\mu \nu}(\mathbf{x}),
$$

then we attain separation of variables in the Schrödinger equation.

If we denote the constant of separation by $E$, the equation for $T\left(x^{4}\right)$ is

$$
\partial_{4} T\left(x^{4}\right)+\mathrm{i} E T\left(x^{4}\right)=0 .
$$

Its solution is

$$
T\left(x^{4}\right)=e^{-\mathrm{i} E x^{4}}
$$

The equation for $X_{\mu \nu}(\mathbf{x})$ is

$$
\nabla^{2} X_{\mu \nu}(\mathbf{x})+2 m E X_{\mu \nu}(\mathbf{x})=0 .
$$

The Fourier transform of this equation is

$$
\int d^{3} p e^{\mathrm{i} \mathbf{p} \cdot \mathbf{x}}\left[-p^{2}+2 m E\right] X_{\mu \nu}(\mathbf{p})=0,
$$

which, based on the independence of the plane waves for each value of $p$, gives the dispersion relation for a non-trivial solution:

$$
E=\frac{p^{2}}{2 m}
$$


This is the non-relativistic energy of a free-particle, as expected, since we have chosen $T_{\mu \nu}=0$.

With the dispersion relation, Eq. (25), the solution of Eq. (24) is given by an amplitude $A_{\mu \nu}$ times the plane wave:

$$
X_{\mu \nu}(\mathbf{x})=A_{\mu \nu} e^{i \mathbf{p} \cdot \mathbf{x}} .
$$

The equation for $X_{\mu \nu}(\mathbf{x})$ is solved in the particular gauge given in Section 2. that is, $\hat{h}_{i 4}=\hat{h}_{44}=\hat{h}_{45}=\hat{h}=0$. This leads to

$$
X_{4 i}=X_{44}=X_{45}=0
$$

and

$$
\begin{aligned}
\nabla^{2} X_{i j}(\mathbf{x})+2 m E X_{i j}(\mathbf{x}) & =0 \\
\nabla^{2} X_{i 5}(\mathbf{x})+2 m E X_{i 5}(\mathbf{x}) & =0 \\
\nabla^{2} X_{55}(\mathbf{x})+2 m E X_{55}(\mathbf{x}) & =0
\end{aligned}
$$

The first equation above can be solved in the transverse-traceless ansatz, usual in the $(3+1)$-dimensional spacetime.

The Fourier transform of the Lorenz-like gauge condition leads to

$$
\begin{aligned}
& \mathrm{i} p_{i} \hat{h}^{i 4}-\mathrm{i} E \hat{h}^{44}=0, \\
& \mathrm{i} p_{i} \hat{h}^{i j}-\mathrm{i} E \hat{h}^{4 j}=0 .
\end{aligned}
$$

Then, we have

$$
\begin{aligned}
& \hat{h}^{44}=\frac{p_{i} \hat{h}^{i 4}}{E}, \\
& \hat{h}^{4 j}=\frac{p_{i} \hat{h}^{i j}}{E} .
\end{aligned}
$$

By substituting the second equation into the first one, we obtain $\hat{h}^{44}$ in terms of $\hat{h}^{i j}$ :

$$
\hat{h}^{44}=\frac{p_{i} p_{j} \hat{h}^{j i}}{E^{2}} .
$$

Consider a single plane wave; that is, consider a direction of propagation

$$
\mathbf{n}=\frac{\mathbf{p}}{|\mathbf{p}|}
$$

Furthermore take the direction of propagation along the $z$-axis; that is,

$$
p_{i}=p \delta_{i 3} \text {. }
$$

With this restriction, some simplifications follow:

$$
\hat{h}^{44}=\frac{p^{2}}{E^{2}} \hat{h}^{33},
$$


and

$$
\hat{h}^{4 j}=\frac{p}{E} \hat{h}^{3 j} .
$$

Together with the condition,

$$
\hat{h}=\hat{h}_{11}+\hat{h}_{22}+\hat{h}_{33}=0,
$$

this gives

$$
\left(\hat{h}_{\mu \nu}^{T T}\right)=\left(\begin{array}{ccccc}
\hat{h}_{11} & \hat{h}_{12} & \hat{h}_{13} & 0 & \frac{p}{E} \hat{h}_{13} \\
\hat{h}_{12} & -\left(\hat{h}_{11}+\hat{h}_{33}\right) & \hat{h}_{23} & 0 & \frac{p}{E} \hat{h}_{23} \\
\hat{h}_{13} & \hat{h}_{23} & \hat{h}_{33} & 0 & \frac{p}{E} \hat{h}_{33} \\
0 & 0 & 0 & 0 & 0 \\
\frac{p}{E} \hat{h}_{13} & \frac{p}{E} \hat{h}_{23} & \frac{p}{E} \hat{h}_{33} & 0 & \frac{p^{2}}{E^{2}} \hat{h}_{33}
\end{array}\right) .
$$

Note that Eq. (26) is also used in the standard relativistic treatment and it is called "traceless condition" in that relativistic context. The label TT, attached to $\hat{h}_{\mu \nu}$, indicates that we have chosen the Galilean analogue of the transverse-traceless gauge. In terms of the amplitudes, we obtain

$\hat{h}_{\mu \nu}^{T T}\left(x^{5}, x^{4}, z\right)=\left(\begin{array}{ccccc}A_{11} & A_{12} & A_{13} & 0 & \frac{p}{E} A_{13} \\ A_{12} & -\left(A_{11}+A_{33}\right) & A_{23} & 0 & \frac{p}{E} A_{23} \\ A_{13} & A_{23} & A_{33} & 0 & \frac{p}{E} A_{33} \\ 0 & 0 & 0 & 0 & 0 \\ \frac{p}{E} A_{13} & \frac{p}{E} A_{23} & \frac{p}{E} A_{33} & 0 & \frac{p^{2}}{E^{2}} A_{33}\end{array}\right) e^{-\mathrm{i}\left(m x^{5}+E x^{4}-p z\right) .}$

This is a wave-type solution for the Schrödinger-type equation in the transversetraceless gauge.

This suggest the following identifications:

$$
\omega=E, \quad \text { and } \quad k=\frac{2 \pi}{\lambda}=p,
$$

with phase velocity

$$
v=\frac{\omega}{k}=\frac{E}{p} .
$$

This result is also obtained in the usual quantum mechanics, described by the Schrödinger equation.

The analogue of Eq. (27), in the usual four-dimensional GR, exhibits only two degrees of freedom. We can reduce our five degrees of freedom to only two independent entries by setting

$$
A_{13}=A_{23}=A_{33}=0,
$$


in which case only $A_{11}$ and $A_{12}$ will remain in the matrix $\hat{h}_{\mu \nu}^{T T}$.

The meaning of the extra components in the 5-dimensional spacetime is still to be clarified. We remark that the additional degrees of freedom are associated to the massive character of the weak field solution, manifested by the dependence on the $x^{5}$-coordinate.

\subsection{Diffusion equation}

Now we analyze an ansatz analogous to the previous one, but avoiding the complexification. The gravitational field is still supposed to be massive, since the dependence with the fifth-coordinate is kept. If $\hat{h}_{\mu \nu}$ is chosen as follows,

$$
\hat{h}_{\mu \nu}=e^{-m x^{5}} \hat{\mathfrak{h}}_{\mu \nu}\left(\mathbf{x}, x^{4}\right),
$$

then Eq. (14) becomes an inhomogeneous diffusion equation,

$$
-2 \kappa T_{\mu \nu}=\nabla^{2} \hat{h}_{\mu \nu}+2 m \partial_{4} \hat{h}_{\mu \nu}
$$

with $-\frac{1}{2 m}$ being the diffusion coefficient. Note that Eq. (19), substituted into Eq. (30) produces an equation similar to Eq. (20) but with an additional time-derivative of $\hat{h}_{55}$, which is expressed in terms of $\phi$ by integrating Eq. (19).

Notice that Eq. (30) is obtained when a gauge is fixed. If we suppose that the gauge is not fixed and that the field is static, i.e. $\hat{\mathfrak{h}}_{\mu \nu}\left(\mathbf{x}, x^{4}\right)=\hat{\mathfrak{h}}_{\mu \nu}(\mathbf{x})$, then, from Eqs. (13) and (29), we find

$$
\begin{aligned}
\kappa T_{44} & =-\frac{1}{2} \nabla^{2} \hat{h}_{44}, \\
\kappa T_{55} & =-\frac{1}{2}\left(2 m \partial_{i} \hat{h}_{5}^{i}+\nabla^{2} \hat{h}_{55}\right), \\
\kappa T_{45} & =\frac{1}{2}\left(m \partial_{i} \hat{h}_{4}{ }^{i}+\nabla^{2} \hat{h}_{45}\right) .
\end{aligned}
$$

The equations in which $\partial_{i} \hat{h}_{4}{ }^{i}$ and $\partial_{i} \hat{h}_{5}{ }^{i}$ are non-zero constants reproduce the Poisson equation with extra terms analogous to the cosmological constant. This particular choice leads to a Newton-Hooke force; that is, a Newtonian potential plus a (linear) Hooke-like contribution [19], showing that the gravitational constant should be related to the mass of the gravitational field.

Note that it is not possible to obtain such an equation in four-dimensional GR. In principle, it could be done in five-dimensional GR, but this would be far more artificial than the Galilean context utilized here. Further comments are in Section 4 . 


\section{Concluding remarks}

In this paper, we have retraced the procedures normally leading to the investigation of gravitational waves in GR, but we have replaced the Lorentz metric with the Galilean metric, Eq. (3), so that the gravitational theory considered is locally Galilean invariant. The reason for doing this is that the Galilei group underlies the appropriate kinematics for low-energy, or nonrelativistic, phenomena; therefore, the Galilean symmetry must be taken into account when non-relativistic objects are causing the gravitational perturbations.

In this paper, we examined the weak-field approximation with local Galilean invariance with a $(4+1)$-dimensional Minkowski manifold which leads to Galilei-invariant equations, when the physical quantities are properly introduced. While there exist 5-dimensional models of GR which have no connection with Galilean symmetry, there are a few reasons to consider Galilean gravity. For instance, the definition given in Eq. (19), even though permitted in five-dimensional GR, is better justified in the Galilean context because Eq. (17) associates the mass density to the component $T_{55}$ of the energy-momentum tensor. Another feature that distinguishes 5-dimensional GR and the Galilean formalism employed here is that the ansätze, given in Eqs. (15), (29) and (21), appear in a natural way within the Galilean framework [16], whereas no such motivation occurs in GR .

The three ansätze considered in Section 3 lead to a Poisson equation, a diffusion equation, and a Schrödinger equation. The Poisson equation allows us to introduce the AQUAL field equations which describe the MOND theory. This definition and the real anzatz lead us to suggest a modified version of the AQUAL field equations. Whether this equation will be relevant in cosmology remains to be explored.

\section{Acknowledgments}

We acknowledge partial support by the Natural Sciences and Engineering Research Council (NSERC) of Canada. RRC and PJP acknowledge financial support from $\mathrm{CNPq}$ (Brazil) and thank the Physics department, University of Alberta, for providing the facilities. RRC is grateful to Prof. V P Frolov for the kind hospitality extended to him. The authors would like to thank the referee for useful comments. 


\section{References}

[1] Lévy-Leblond J M 1971 in Group Theory and Applications, vol. 2 Ed. E.M. Loebl (New York: Academic Press) p. 221

[2] Le Bellac M, Lévy-Leblond J M 1973 Nuov. Cim. B 14217

[3] Santos E S, de Montigny M, Khanna F C, and Santana A E 2004 J. Phys. A: Math. Gen. 379771

[4] Lévy-Leblond J M 1967 Comm. Math. Phys. 6286

[5] Takahashi Y 1988 Fortschr. Phys. 3663

Takahashi Y 1988 Fortschr. Phys. 3683

[6] Omote M, Kamefuchi S, Takahashi T, and Ohnuki Y 1989 Fortschr. Phys. 37933

[7] Soper D E 1976 Classical Field Theory (Wiley and Sons: New York) Sect. 7.3

Pinski G 1968 J. Math. Phys 91927

Duval C, Burdet G, Künzle H P, and Perrin M 1985 Phys. Rev. D 31 1841

Duval C, Gibbons G W, and Horváthy P 1991 Phys. Rev. D 433907

Künzle H P and Duval C 1994 in 'Semantical Aspects of Spacetime Theories' (U. Majer and H.J. Schmidt, Eds.) p. 113, BIWissenschaftsverlag, Mannheim

Kapuścik E 1986 Acta Phys. Pol. B 17569

[8] Cuzinatto R R, Pompeia P J, de Montigny M, Khanna F C 2009 Schwarzschild-type solution in an effective gravitational theory with local Galilean invariance. Available at: Arxiv: 0903.2488

[9] Weinberg S 2008 Cosmology Oxford, New York

[10] Milgrom M 1983 Astrophys. J. 270365

Milgrom M 1983 Astrophys. J. 270371

Milgrom M 1983 Astrophys. J. 270384 
[11] Bekenstein J D and Sanders R H 2005 in Mass Profiles and Shapes of Cosmological Structures G. Mamon, F. Combes, C. Deffayet, B. Fort (Eds.), EAS Publications Series. Available at: ArXiv: astro-ph/0509519

[12] Bekenstein J D 2004 Phys. Rev. D 70083509

[13] Bekenstein J 2006 Contemp. Phys. 47387

[14] Weinberg S 1972 Gravitation and Cosmology: Principles and Applications of the General Theory of Relativity John Wiley and Sons, New York

[15] De Sabbata V and Gasperini M 1985 Introduction to Gravitation World Scientific Publishing Co Pte Ltd, Singapore

[16] Santos E S, de Montigny M and Khanna F C 2005 Ann. Phys. (NY) 32021

Abreu L M, de Montigny M, Khanna F C and Santana A E 2003 Ann. Phys. (NY) 308244

[17] de Montigny M, Khanna F C and Saradzhev 2008 Ann. Phys. (NY) 3231191

[18] Gregory R and Laflamme R 1993 Phys. Rev. Lett. 702837

[19] Aldrovandi R, Barbosa A L, Crispino L C B and Pereira J G 1999 Class. Quant. Grav. 16495 